\title{
Omnidirectional Video in Museums - Authentic, Immersive and Entertaining
}

\author{
Jaakko Hakulinen $^{[0000-0001-8054-7265]^{*}}$, Tuuli Keskinen ${ }^{[0000-0002-0170-5016]}$, Ville Mä- \\ kelä ${ }^{[0000-0001-6095-2570]}$, Santeri Saarinen ${ }^{\text {[0000-0003-3014-6177] }}$, and Markku Turunen ${ }^{\text {[0000-0001- }}$ \\ 7395-0769] \\ University of Tampere, Tampere, Finland \\ \{jaakko.hakulinen, tuuli.keskinen, ville.mi.makela, \\ santeri.saarinen, markku.turunen\}@uta.fi
}

\begin{abstract}
Omnidirectional video (ODV) provides possibilities for capture and presentation of cultural content for museums and other exhibitions. $360^{\circ}$ video capture can record authentic environments and activities, and enables immersive viewing of such material. We present the potential of ODV by reporting the learnings from long-term user experience data collection with two ODV-based museum installations; a head mounted display (HMD) based rally simulator, and a road grader simulator utilizing three projectors and haptic feedback. The results show the value of immersion, provide insights into the possibilities and challenges of interactivity, and show, how haptic feedback can improve the overall experience. The results also provide support to the use of physical elements in museum installations. In addition, we managed to avoid cyber sickness, a common problem with immersive VR content, in the installations. Overall, the respondents strongly support the conception that these kinds of installations would make museums more desirable places to visit.
\end{abstract}

Keywords: omnidirectional video, $360^{\circ}$ video, museum installation, user experience, immersion, haptic feedback, head mounted display.

\section{Introduction}

$360^{\circ}$ video, aka omnidirectional video (ODV) provides possibilities for the capture and presentation of cultural content for museums and other exhibitions. Video can be used to capture authentic places, environments and activities, and full spherical $360^{\circ}$ capture enables immersive viewing of such material. The limitation is that ODV is still static video in spherical form, and therefore ways to interact with it are restricted. Still, within the restrictions, many kinds of interactions are possible. Compared to modeling 3D graphics, ODV capture is cost efficient and it ensures authenticity of the resulting material and thus can also have significant cultural-historical value. To produce $360^{\circ}$ video, the captured place or activity needs to be physically accessed. However, video can often be captured in locations and during activities, where most museum goers 
would not be able to go to or join in real life. It is also possible to capture historic events which cannot be experienced any other time.

In many cases, ODV is viewed with what can be considered immersive setups. Headmounted displays (HMD) are the most common and most immersive. Still, CAVE type solutions utilizing multiple projectors or displays can also reach significant levels of immersion [12]. Both share the fundamental interaction where content can be observed naturally by turning one's head towards a point of interest - whether it be accessed through the viewport of a HMD, or observed in a CAVE via the surrounding displays. The resulting immersion can lead to interesting and engaging experiences and is therefore often desirable.

In the context of museums, $360^{\circ}$ video can be valuable for several reasons. For visitors, it can provide engaging experiences, which can attract more people. The engagement can also have pedagogical value as engaged visitors may absorb more information. When visitors are allowed to choose what part of the omnidirectional video they are looking at, different visitors may also focus on details that are particularly interesting to them. ODV can also provide means to record culturally significant environments and experiences in an efficient and comprehensive manner.

To study the possibilities of ODV in the context of museums we created two installations to automobile and road museum Mobilia (Kangasala, Finland); a simulator of a vintage road grader, followed by a rally simulator. These utilize ODV in different ways. The HMD-based rally simulator provides a more common approach with minimal interaction but the viewing takes place inside an actual rally car. In contrast, the road grader simulator features a rough physical replica of a grader interior, utilizes three projectors, and provides more interactivity (compared to the rally simulator) as the system reacts to pedals and a steering wheel. Both installations utilize audio (vehicle sounds, speech), and the grader simulator also features haptic feedback in the simulator's seat.

In this paper, we look at the subjective feedback collected with several iterations of the installations. This work provides understanding on the effects of additional modalities, different levels of immersion, and different levels of interactivity on user experience. The work considers also the target user group in the museum which includes children in great proportion. Feedback for the four versions of the road grader was gathered during a period of two years starting from June 2015, and feedback for the rally simulator was gathered for one year starting from June 2016. The results illustrate the value of immersive viewing and show how haptic feedback can improve the overall experience. We also present effects of different interaction metaphors. Finally, we discuss how cyber sickness can be avoided, as demonstrated by our HMD-based rally simulator installation.

Next, we will provide background on the utilization of ODV in interactive installations. Chapters 3 and 4 describe our installations and the feedback we have collected during their deployment in the museum environment. Chapter 5 summarizes and discusses our experiences and findings, focusing on immersion, interactivity and the potential of additional modalities. We believe these concepts are critical in understanding the potential of ODV. The paper is closed with conclusions in Chapter 6. 


\section{Related Work}

ODVs have been used in many domains and contexts. Remote operations and telepresence applications were the first serious applications [5, 7, 23, 37], therapeutic $[9,26,30]$ and education [10] solutions have also been studied and consumer products have followed recently. Many of these applications offer interactive content and have UI elements which are often crucial features for pleasant user experience [4, 30]. Different interaction techniques in interactive omnidirectional video (iODV) [12] applications have also been studied. Desktop based interfaces [20] exists and most recent work has focused on HMD-based interaction, where head orientation and dwell-time based interaction is common [12]. Gesture-based interaction [2, 24 27, 31, 39] and secondscreen interfaces [38] have been studied as well. ODV has also been used to simulate AR interactions [3]. Some applications have utilized also additional modalities [28]. Argyriou et al. [1] as well as Saarinen et al. [32] have presented guidelines for omnidirectional videos in their research.

Simulators and other interactive installations in museums form one potential application of ODV. It has been utilized in various settings with historical and cultural content. Cultural heritage has been recorded and displayed [16, 17, 22], and various performances and events have been captured [e.g., 6, 18] and even used as part of theatrical experiences [8].

The application of ODVs usually relies on immersive approach. Immersion and (the feeling of) presence have been given various definitions by different authors. Slater [33] separates the two so that immersion is purely objective measure defined by rendering software and display technology. Related parameters include display field of view (FOV), field of regard (FOR), resolution, update frequency, and support for stereoscopy. In contrast, presence is "an individual and context-dependent user response" to using a system, an individual's experience of 'being there'. In this paper, we focus mostly on the objective immersion per Slater's definition, but also inquire about users' subjective views on feeling involved. A significant challenge with immersive virtual content consumption, including ODV content is that some users experience cyber sickness [29] as the result. This can be battled with well-designed content and interaction.

In immersive virtual reality, ODV can be contrasted to 3D content created either by modeling, utilizing existing CAD models or by capturing objects and environments, for example, with photogrammetry or laser scanning. Creating 3D content is in most cases a time-consuming process which requires high expertise. In contrast, capturing ODVs is possible with little training, in particular with the latest consumer grade cameras. The ODV captures a lot of detail, and where the virtual content should carefully match some real environment, ODVs can be particularly valuable.

ODV can be viewed in different ways. The recent advances in VR technology have made head mounted displays (HMD) the most discussed way but options range from viewing with regular computers (e.g., YouTube, Vimeo and Facebook support $360^{\circ}$ video) to hand-held devices with magic lens style interaction and CAVE like environments consisting of multiple projectors or multiple large displays. From the interaction point of view, most ODV viewing setups allow users to control the viewing direction, be it by head orientation in HMD, device orientation with a hand-held mobile phone, 
or with mouse on desktop use. The weakness of ODVs is that it limits the user's movement in the virtual world. The user can only move between separate videos, unlike with full 3D content, where free exploration can be enabled. However, there are other forms of interaction possible with ODV content, which can be combined in novel ways. In addition to the traditional video controls (playback rate and position) [20], ODV allows different viewing projections which can create interesting effects. For example, so called small-planet projection (stereographic projection) creates an interesting view into videos, particularly to videos captured while the camera is moving.

While the array of ODVs applications found in literature provides many ideas and illustrations of the possibilities, the evaluations have been small-scale and mostly done in laboratories. As technology now allows real-life application of ODV-based solutions, there is need to validate the value of these solutions with real users and in real context. In the following, we present the long-term evaluations of two ODV-based museum installations and an analysis of feedback collected over several iterations. The results provide insights into aspects of such installations valued by museum visitors.

\section{Road Grader Simulator}

We looked at the possibilities of creating an interactive ODV based installation by building a road grader installation. The system is a simulator of a vintage road grader based on ODV, audio, and haptic feedback. It is targeted especially for families with children, including grandparents with their grandchildren. This target user group was specified by the museum personnel. An approach with a physical steering wheel and pedals was chosen since the target user group, according to the museum staff, values activities which resemble physical driving. The simulator is installed in a dedicated, darkened room and is part of the main exhibition of the museum. The simulator has been available for visitors since June 2015 and over the two years we have updated the system three times, resulting in four different iterations. Subjective feedback has been collected with a questionnaire the whole time and the collected feedback has both guided our development steps and provided data on the effects of these updates. These results are presented per iteration and discussed in the following. We start by describing the technical setups, and the system's evaluation procedure along with the findings.

\subsection{System Description}

The content of the road grader installation is based on $4 \mathrm{~K}$ resolution $(3840 * 1920$ pixels, 25 frames per second) ODV. Although using videos makes it impossible to allow users to drive around freely, ODV was chosen because it is more cost-efficient to produce than 3D models, ensures the authenticity of the content and a real historical grader was available for capturing realistic material. A camera setup capable of capturing full spherical video was attached on the roof of the grader and a microphone was placed inside the cabin while the grader was driven around on small roads.

The current system setup of the simulator can be seen in Figure 1. It consists of a large display (three full-HD projectors with picture area of about $6 * 1.5$ meters), a seat 
with haptic feedback, a steering wheel and brake and accelerator pedals, a computer, an audio speaker, and a set of sensors. The sensors are a 3D accelerometer and gyroscope for the steering wheel (Yocto3D by YoctoPuse ${ }^{1}$ ), linear potentiometers for the pedals (connected via a YoctoKnob), and a light sensor looking through a hole in the seat (YoctoLight) to detect whether someone is sitting on it. The setup is mechanically robust since the visitors cannot apply force directly to the sensors. The seat has a device called Buttkicker ${ }^{2}$ to provide strong vibration feedback.

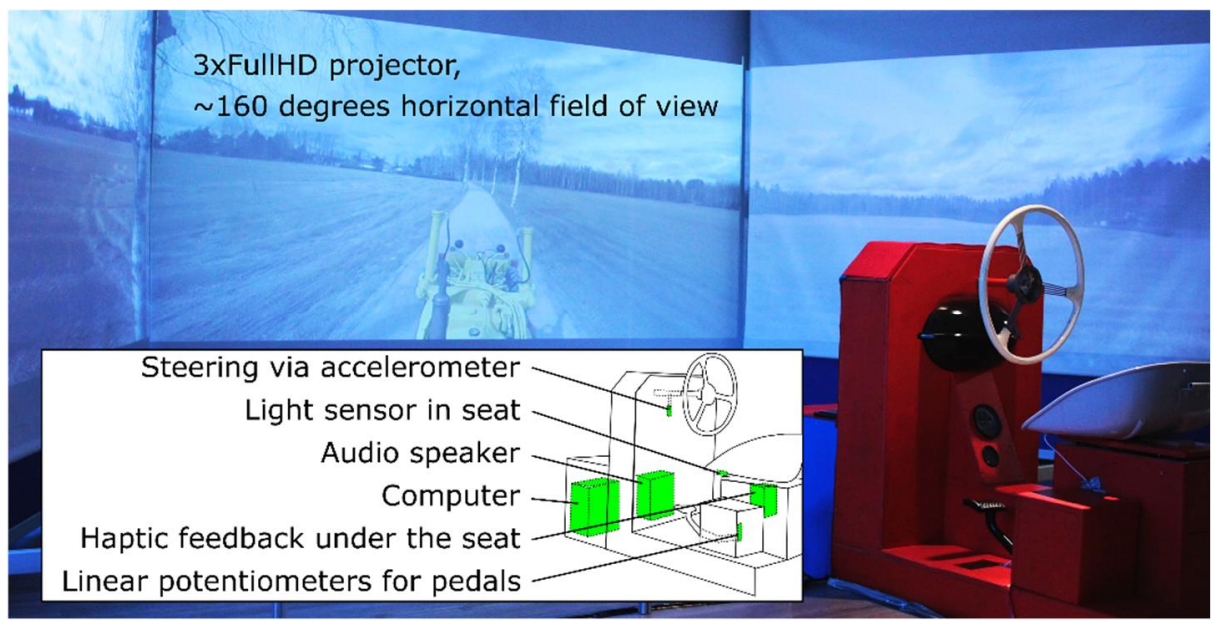

Fig. 1. The road grader simulator setup.

The display setup originally consisted of a single full-HD projector covering $\sim 53^{\circ}$ of the driver's field of view. This was later upgraded to three projectors whose image covers about $160^{\circ}$ of the driver's horizontal field of view with the total horizontal resolution of 5760 pixels, which is higher than the resolution of the utilized video material. The display surfaces are flat and there are clearly visible edges between the three projection surfaces. The distance from driver's seat to the projection screens is roughly 2.5 meters. The room is dimmed so that very little outside light comes in to ensure good contrast in the projected picture. The driver's seat is lit with a spotlight hanging from ceiling.

The steering wheel movement is limited to roughly 65 degrees per direction so a single accelerometer can tell the steering angle. This is a mechanically simple solution with minimal moving parts. In the first version, the steering wheel rotation was unlimited and used a pulse counter rotated via a set of gears. The update was made because user feedback indicated that the initial solution did not provide good experience, and the implementation was prone to fail. For the third version we also updated the software so that a more distinct visual response to accelerating and braking is provided.

1 http://www.yoctopuce.com/

2 http://www.thebuttkicker.com/ 
The pedals and their mounting are from an old utility vehicle and therefore very sturdy. The linear potentiometers sensing the pedal movements are attached to the mounting and hidden inside the encasing of the system.

The haptic feedback on the seat utilizes a low frequency audio transducer which physically rumbles the seat in vertical direction based on audio signal. The audio is the same the users hear, consisting of engine sounds and various other noises the grader makes. The seat is mounted on rubber feet to allow movement. The resulting effect resembles, to reasonable extent, the rumble of the grader running on a dirt road.

The light sensor in the seat detects if somebody is sitting, and it is placed under a small hole near the front edge. There is a spotlight illuminating the seat, which ensures that the light sensor gets enough light in the darkened room when nobody is sitting down, and communicates to the visitors where they should go in the room. The only problem with the light sensor solution was that initially it was located too far back so that small children did not cover the sensor when sitting down. This was problematic since small children are in the target user group of the installation.

The computing hardware and software consists of PC computer running an HTMLbased front end application, which displays the video and plays back audio, and a back end which acts as an http-server, reads the sensors and serves their data to the front end.

Interactivity. The system plays back the ODV and related audio. There are several interactive elements.

Sitting down. When nobody is sitting, the video plays back in a so-called small planet projection. When somebody sits down, the video animates in three seconds into a view close to first person perspective (the camera was placed on the roof of the grader so the viewpoint is somewhat higher than in real life and vehicle interior is not visible).

The break and acceleration. The pedals control a simple simulation of driving speed and the related engine rpm. No gear changes were included in the simulation and the grader is simulated so that it never comes to complete halt, even if break is fully depressed. The speed is communicated in three different ways.

First, video playback rate is varied. There are two rates, 0.5 when the user is braking and 1.0 times original rate otherwise. The rate is not mapped directly to the virtual vehicle speed as playback of the high resolution (4K) video at random rates is unreliable. As people are not very good at detecting minor changes in video playback rate, this solution has not resulted in any negative feedback.

Second, audio matches the simulated engine RPM. From the recordings, sound clips with different constant RPM values and samples with increasing and decreasing RPM were selected. Based on the simulated engine RPM, appropriate samples are selected and played back with varying playback rate (rate adjustment changing the pitch of the sound to match the exact RPM). The software selects the sample, which matches the current situation with minimal change from the original sampling rate to create realistic vehicle sounds. The audio is both played back via the audio speaker and fed to the Buttkicker to create haptic feedback. 
Third, video projection changes somewhat based on the speed. With the ODV both viewing direction and the field of view can be adjusted. The field of view of the central projector varies so that high speed has narrower field of view than slow speed (the average FOV is 60 degrees and it can change \pm 15 degrees). This effect emphasizes the appearance of motion even when the video playback speed does not change. In addition, the camera view tilts so that in fast speed the camera looks slightly downwards, displaying more road near the vehicle while slow speed shows more sky, further affecting the experienced speed.

Steering. Turning the steering wheel adjusts a set of visual parameters to provide some feeling of steering. Camera heading adjusts so that steering left turns the camera left and vice versa (i.e., like in a real vehicle). User comments indicated that some people would assume the camera to turn the other way and we experimented with both mappings. The maximum view rotation is about $\pm 30^{\circ}$. We also tried rolling the camera sideways but this effect was considered potentially nauseating and was never included in the production versions. The virtual camera can also move sideways inside the video sphere creating some distortion somewhat similar to sideways movement. This effect is used to small extent, the maximum offset being $0.06 *$ video sphere radius.

The iterations. The four main versions and the changes between them can be seen in Table 1.

Table 1. Summary of the different versions of the road grader simulator

\begin{tabular}{|l|l|l|}
\hline Version & Main features & $\begin{array}{l}\text { Main changes compared to the ear- } \\
\text { lier version }\end{array}$ \\
\hline $\begin{array}{l}\text { ": } \\
\text { "baseline" }\end{array}$ & $\begin{array}{l}\text { 1 projector }\left(\sim 53^{\circ} \mathrm{FOV}\right) \\
\text { unlimited steering wheel rotation }\end{array}$ & - \\
\hline $\begin{array}{l}2: \\
\text { "haptic" }\end{array}$ & $\begin{array}{l}\text { projector, haptic feedback } \\
\text { 3: }\end{array}$ & $\begin{array}{l}\text { Haptic feedback (Buttkicker) in- } \\
\text { stalled }\end{array}$ \\
\hline $\begin{array}{l}\text { "3 screens" } \\
\text { "inverted } \\
\text { steering" }\end{array}$ & $\begin{array}{l}3 \text { projectors }\left(\sim 160^{\circ} \text { FOV }\right), \text { haptic } \\
\text { feedback, steering limited to } \pm 65^{\circ}\end{array}$ & $\begin{array}{l}\text { Added projectors, steering wheel ro- } \\
\text { tation limited }\end{array}$ \\
\hline
\end{tabular}

\subsection{Evaluation}

We wanted to understand the user experience the installation provides and monitor, how the upgrades change it. Therefore, we collected subjective feedback from users with a questionnaire. With the data over the four iterations, we can also look at the roles of different elements in the experience. The long-term evaluation presented here covers data from altogether 215 respondents received between mid-June 2015 and early-June 2017. 
Subjective Data Collection. User experiences were collected with a paper-form questionnaire. Because a suitable questionnaire was not readily available, one was created to correspond with the context, and the objectives of the system. The custom questionnaire was required partly due to the target user group. We needed a questionnaire, which children, possibly with the help of their parents can fill in without guidance. This required simple phrasing and limited length, making most existing questionnaires, especially those focused on immersion and presence, unsuitable. We also wanted to cover aspects specific to the content. Thus, in addition to more general user experience aspects the questionnaire inquired about immersion and the appeal of the system in the context of a (automobile) museum. The questionnaire design was based on our earlier user experience evaluations and questionnaire development [e.g., 15, 35], including our earlier museum installation evaluations [34].

The questionnaire includes ten statements with a five-step rating scale on the range of Totally disagree - Neither agree or disagree - Totally agree. The statements can be seen in Figure 2. In addition, the overall liking of the system was inquired with a fivestep smiley face scale ranging between extremely unhappy (1) - extremely happy (5). To support and explain the quantitative results, the questionnaire includes open-ended sentences inquiring the best and worst features of driving the road grader, as well as other comments considering the driving or the simulator. The questionnaire ends with a background information section asking the respondent's age, gender (Boy/male, Girl/female, Other), personal interest towards technology (e.g., cars), gaming and history as statements rated on a similar disagree-agree scale as the user experience statements.

A table for filling in the questionnaire, and a return box, are situated in the vicinity of the simulator in the same room. Although an electronic questionnaire would have reduced the required work, a paper-form was selected to avoid equipment maintenance and to ensure approachable feedback mechanism for non-technical museum visitors such as older people with their grandchildren. In order to motivate people to provide feedback small product prizes have been raffled amongst the respondents who have provided their contact information.

Respondents. Between mid-June 2015 and early-June 2017, i.e., concerning all four versions of the road grader simulator, we received feedback from altogether 215 respondents (103 boy/male, $66 \mathrm{girl} / \mathrm{female}, 4$ other, $42 \mathrm{did}$ not answer). The ages of the respondents varied between $1.5-68$ years (mean $=22.9, \mathrm{SD}=17.9)$, the age group of 0 10 -year-olds covering about 38\%, 11-20-year-olds about 19\%, 21-35-year-olds about $15 \%, 36-50$-year-olds about $18 \%, 51-64$-year-olds about $9 \%$, and 65 -year-olds or older only about $2 \%$ of the responses. The respondents were very interested in technology (median 5/5), and somewhat interested in gaming and history (median 4/5).

\subsection{Results}

A summary of the user experience statement responses for the different versions is presented in Figure 2. As can be seen from the statement results, already the first version 
received good ratings from the users. They totally agreed that the first impression of the simulator was interesting and that these kinds of simulators would increase their interest in museum visits. Along with the experience in general, respondents mentioned, e.g., the possibility to turn the steering wheel and to push the pedals as the most positive things about the simulator. This indicates that people, especially children, liked the concrete installation which allowed them to do tangible actions. However, one not being able to actually steer the virtual vehicle raised critique in one third of the feedback. This can be seen also in the median experience of $2 / 5$ for the statement "I felt like I was actually steering a road grader". Although we would not be able to allow free driving with this kind of a setup, the illusion of one steering him-/herself was probably weakened by the fact that in the first version, the rotation of the steering wheel was not limited but the wheel rotated endlessly to both directions. The simulator did not feel realistic enough, and the median overall liking for the first version of the road grader simulator remained on a neutral level, as can be seen in Figure 3.

Our next step was to install the Buttkicker to the seat in order to enrich the experience through haptic feedback. Compared to the previous version, this addition did improve the experiences statistically significantly (Mann-Whitney $\mathrm{U}, \mathrm{p}<0.05$ ) in regards to the irritatingness and the fun of driving, the willingness to drive again, and the overall liking of the road grader simulator. In the open questions, $18 \%$ of the best thing answers explicitly mentioned the haptic feedback as the best things. Considering the worst thing answers, $29 \%$ of the mentions were either nothing or the respondent marked a "-“ indicating he/she could not come up with negative aspects, whereas the same proportion was only $2 \%$ for the first version.

Unsurprisingly, the addition of haptic feedback did not help with the fact that the respondents kept mentioning the lack of steering in their feedback. Thus, for the third version of the road grader simulator, we tried to improve the feel of the steering wheel by limiting its rotation to \pm 65 degrees, instead of the unlimited rotation. In addition, and most importantly, we installed three projection screens for the simulator expanding the horizontal field of view from about 53 degrees to about 160 degrees. After these changes, the proportion of positive mentions about the scenery and pictures increased, and the respondents felt slightly stronger as if they would have been actually aboard a grader. However, there were no statistically significant (Mann-Whitney U) differences in the experiences compared to the previous version with haptic feedback addition. This was a rather unexpected result. It may be that the screens are too far away from the user, and as the installation space is still rather large, expanding the horizontal field of view was not enough to enable the feeling of immersion: the ceiling, the floor and the walls on extreme left and right of the user were still visible. 


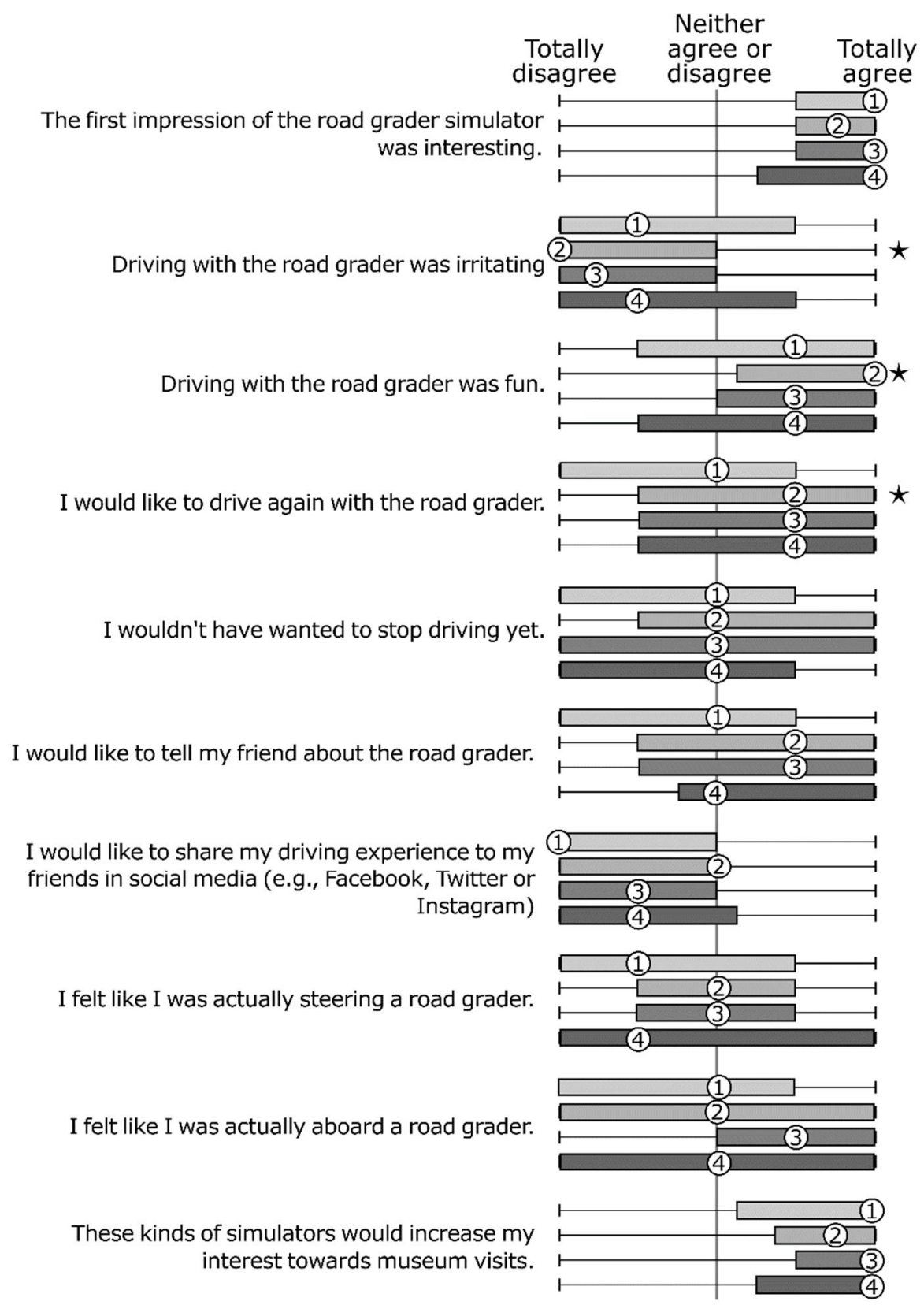

Fig. 2. Reported user experiences about the road grader simulator. The numbered circles represent the median experience values of the different versions: 1) baseline version $(n=63-72), 2)$ version with haptic feedback $(n=29-32), 3)$ version with three screens $(n=57-62)$, and 4) version with camera turning to the opposite as the steering wheel $(n=32-37)$. The whiskers indicate the minimum and maximum responses, and the gray boxes indicate the interquartile range. The stars indicate statistically significant differences compared to the previous version. 
How much did you like the road grader simulator overall?

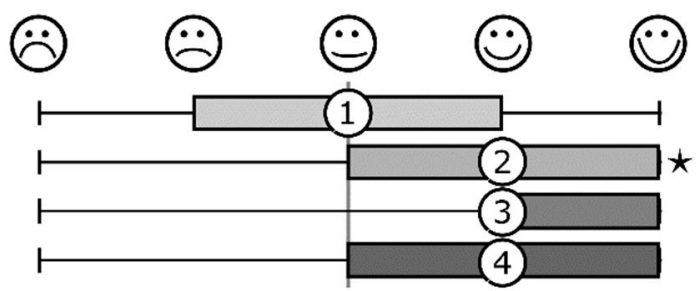

Fig. 3. The overall likings of the four versions of the road grader simulator reported on a smiley face scale. The numbered circles represent the median values of the different versions: 1) baseline version $(n=72), 2)$ version with haptic feedback $(n=30), 3)$ version with three screens $(n=62)$, and 4) version with camera turning to the opposite as the steering wheel $(n=36)$. The whiskers indicate the minimum and maximum responses, and the gray boxes indicate the interquartile range. The star indicates statistically significant difference compared to the previous version.

People have different expectations on how a virtual environment should react to turning the steering wheel. First, our simulator functioned so that turning the steering wheel to the left turned also the view to the left, i.e., one would see further to the left, like in the real driving situation. In the fourth version, we changed this to the opposite to see whether it would affect the user experiences. Rather surprisingly, changing the camera to turn to the opposite direction as the steering wheel showed no statistically significant changes (Mann-Whitney $U$ ) in the experiences. However, regarding the worst things answers, the proportion of lack of steering mentions doubled from about $30-33 \%$ for the previous three versions to about $61 \%$ for this fourth version. Also, the medians for the statements of people feeling like steering a road grader or being aboard one dropped a step after changing the camera turning logic, although the change was not statistically significant.

Our findings from the iterative development of the road grader simulator show that haptic feedback is important for such a setup combining omnidirectional video and concrete, physical elements - a grader cabin with a seat, steering wheel and pedals in this case. It is impossible to allow free steering for this kind of a simulator. Instead, turning the steering wheel turns the virtual camera, not the vehicle itself. This resulted in critique throughout the feedback by the respondents. In order to provide a richer experience, we are developing a new solutions for more realistically mimicking steering by visual effects. The respondents were also rather neutral considering the statement "Ifelt like I was actually aboard a road grader". One obvious reason for this might be that the camera was situated on top of the roof instead of the cabin when shooting the ODV with the actual road grader. However, the limited field of view of the display and lack of actual grader interior and windows which allowed the users to see the room around them most likely were even more significant factors.

\section{$4 \quad 360^{\circ}$ Rally Simulator}

After experimenting with a projector based system, we wanted to investigate the experiences created by an immersive head mounted display ODV installation in the same 
place (Rally Museum in Mobilia). The system uses ODV recorded inside a rally car during real-world rally driving. The target user group still includes children but the installation is directed more towards adult visitors. The video is shot from the car of a well-known rally driver, and thus, visitors can find value in both the immersive experience and the factual content. The installation, set up inside a real rally car, has been in the Rally Museum since its opening in June 2016, and subjective feedback from one year of use is reported in this article.

\subsection{System Description}

The $360^{\circ}$ rally simulator is a system built for the Samsung Gear VR headset and Samsung mobile phones. It utilizes Oculus Mobile VR SDK for displaying the visual material on Gear VR. The content is 4K (3840* 1920 pixels, 25 frames per second) ODV. The simulator is set up inside an actual rally car which is part of the Mobilia Rally Museum collection. Inside the car, the users are able to sit either on the driver's or the co-driver's seat, and while sitting on the driver's seat, also keep their hands on the steering wheel (although the steering wheel does not control the simulator). The car used in the installation is different and older than the car the videos were recorded in. The simulator application is used by putting on the Gear VR headset and separate headphones. See Figure 4 for the physical setup in the museum.

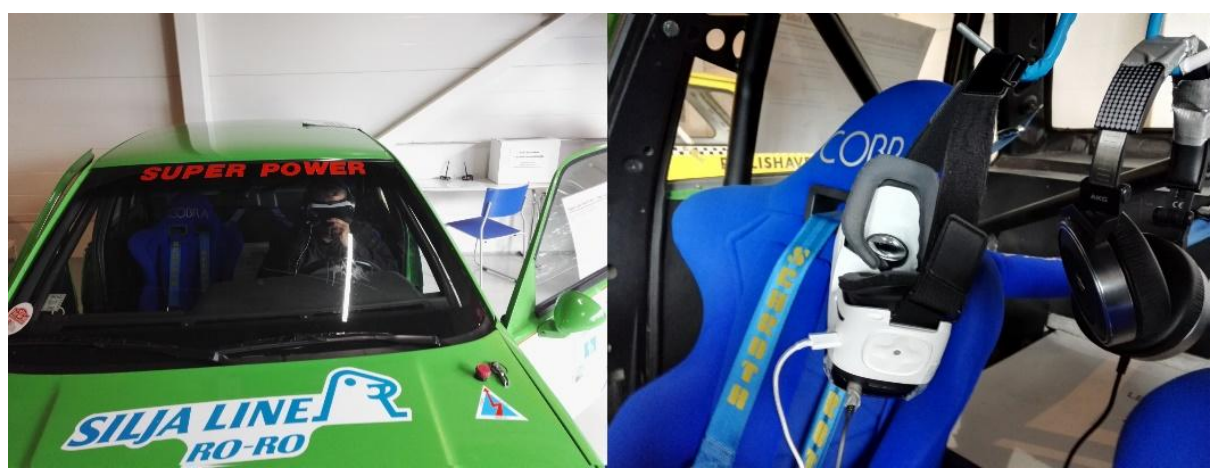

Fig. 4. In the $360^{\circ}$ rally simulator installation, the user is able to sit in a real car and experience a rally stage through VR glasses and headphones.

The application consists of several ODVs and audio recorded inside a rally car during two practice stages by many times national rally champion Juha Salo and his codrivers Marko Salminen and Henri Arpiainen. First, winter-time material with snowy scenery was available for the museum visitors, and for the last four months, it was replaced with material recorded during a summer time practice session. The video materials consist of runs through test tracks and commentary discussion between the driver and co-driver afterwards. The audio consisting of engine sounds and car intercom speech enhances the experience since it allows the users to listen to the pace notes read by the co-driver and hear engine and other sounds. In addition, there is an initial short video from the starting position where the user can activate the driving video by looking 
at an arrow in the front of them. The videos were recorded with the camera placed between the heads of the driver and co-driver so that viewing most of the interior, including pace notes, steering wheel and pedals, is possible. Information about the video (driver, co-driver, car and location) is overlaid on the ceiling of the car with a 3D text element, which is visible when the user looks up with the HMD. This combination of video, audio and actual physical car environment was designed to provide a novel and exciting experience for the visitors.

\subsection{Evaluation}

In order to study museum visitors' experiences with the rally simulator, subjective feedback from the users has been collected with a questionnaire. One of our main goals was to study the effects of a different presentation medium (HMD) on the experience, as opposed to the three-projector setup of the previous simulator. We also wanted to understand how interesting the visitors find the video material without interactivity and how they experience the physical installation. We were also inquiring about cyber sickness as it is a well-known challenge with immersive VR content.

Subjective Data Collection. Similar to the road grader simulator, user experiences of the rally simulator were collected with a paper-form questionnaire. For consistency and comparability reasons, the questionnaire created for the road grader simulator was taken as the basis even though the target user group was somewhat different. In addition to changing the wording to refer to the rally experience, an item inquiring the respondent's physical seat within the car (driver's seat or co-driver's seat) and a statement inquiring whether the simulator caused the respondent nausea were added. Also, the inquiry about the respondent's personal interest in history was replaced with interest in rally racing. The final 11 statements rated on a disagree-agree scale for the rally simulator can be seen in Figure 5.

The questionnaire has been available for rally museum visitors since the simulator's first deployment in June 2016. A table for filling in the questionnaire, and a return box, are situated in the vicinity of the simulator. To motivate people to provide feedback, product prizes have been raffled amongst the respondents of the rally simulator questionnaire as well.

Respondents. Between mid-June 2016 and early-June 2017, we received feedback from altogether 304 respondents (159 boy/male, 103 girl/female, 2 other, 40 did not answer). 256 of these respondents experienced the winter-scenery rally, and for the remaining 48 respondents $(\sim 16 \%)$, the summer-scenery material was presented. The ages of the respondents varied between 4-70 years (mean=32.0, $\mathrm{SD}=17.9$ ), the age group of 0 -10-year-olds covering about 16\%, 11-20-year-olds about 17\%, 21-35-year-olds about 19\%, 36-50-year-olds about 32\%, 51-64-year-olds about 13\%, and 65-year-olds or older only about $3 \%$ of the responses. A clear majority, $94 \%$, of those who reported their physical seat, sat on the driver's seat. The respondents were very interested in 
technology (median 5/5), and somewhat interested in gaming as well as rally racing (median 4/5).

\subsection{Results}

A summary of the user experience statement responses can be seen in Figure 5.

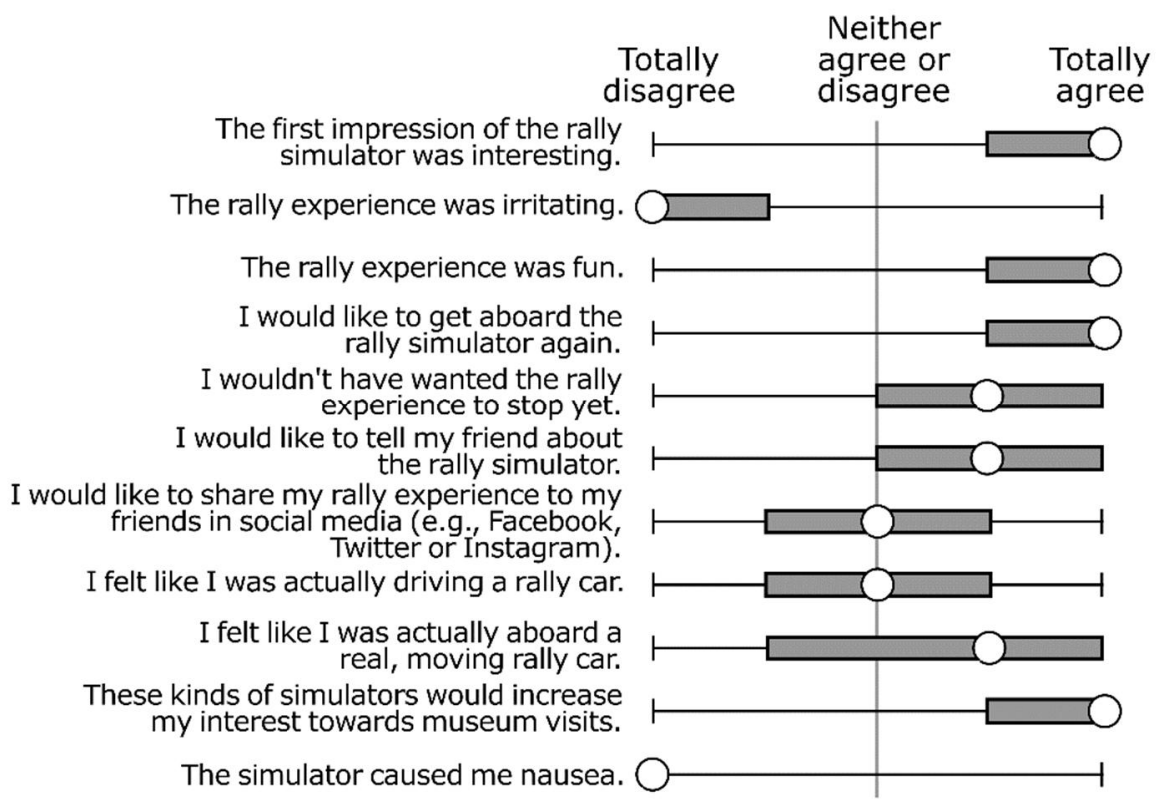

Fig. 5. Reported user experiences with the rally simulator. The whiskers indicate the minimum and maximum responses, the gray boxes indicate the interquartile range, and the white circles represent the median values $(n=273-286)$.

The overall feedback about the simulator has been very positive. For the question "How much did you like the rally simulator overall", the respondents rated their experiences as $4 / 5$ as a median on the smiley face scale. The users have reported the rally experience to be extremely fun (median 5/5), and they would like to get aboard the rally simulator again (median 5/5).

As the simulator did not attempt to mimic the situation of one actually driving a rally car, and the camera was situated between the driver's and the co-driver's seat, the respondents' experiences about the statement "I felt like I was actually driving a rally car" remained neutral (median 3/5). After all, interactivity within the system was limited, and, e.g., the steering wheel and pedals in the physical car did not have on effect on the simulator. However, the respondents somewhat agreed with the statement that they felt like they were actually aboard a real, moving rally car (median 4/5). This is logical, as sitting inside a real rally car during the experience likely increased the sense of "being there". 
As with the road grader simulator, the respondents strongly agreed that these kinds of simulators would increase their interest towards museum visits (median 5/5). An interesting finding is also that the respondents totally disagree with the statement that the simulator caused them nausea (median 1/5), although it is commonly known that $360^{\circ}$ videos experienced with virtual glasses may cause nausea. In fact, a clear majority of $79 \%$ totally disagreed and $10 \%$ somewhat disagreed with the simulator causing them nausea. Only 11 individuals out of the 280 respondents (4\%) totally agreed with the statement.

Considering the open-ended questions, the respondents admired the experience in general, the authenticity, i.e., as if they would have been aboard a real car, and the possibility to sit in a real car. Also, the feeling of speed, the possibilities provided by the ODV, i.e., the ability to look around, and the audio including the pace notes, were mentioned as positive aspects. The negative comments about the rally experience concentrated on the issues related to contrast and lighting of the video material throughout the responses (about $24 \%$ of negative mentions). The videos displayed the car interior properly but the environment outside the car was often overexposed, and the road and its surroundings were hardly visible. In the winter recordings this was mostly due to snowy environment while in the summer recordings (available to the museum visitors since mid-January 2017), direct sunlight created strong contrasts. The material change (from the winter to the summer material) actually decreased some user experience measures (Mann-Whitney $\mathrm{U}, \mathrm{p}<0.05$ ): statements related to first impression, irritatingness and willingness for future use were rated worse.

Overall, the museum visitors obviously have enjoyed our $360^{\circ}$ rally simulator a lot, despite some justified critique: When asking for the worst thing about the rally experience, about $16 \%$ of the mentions explicitly stated there was nothing negative and about $13 \%$ of the mentions were a "-" indicating that those respondents could not come up with negative aspects. Also, when asking free-form comments, about $43 \%$ of the mentions were positive such as "a nice idea", "more of these things", or "a nice addition to a museum".

\section{$5 \quad$ Discussion \& Future Work}

Constructing and iteratively refining the two ODV based museum installations has given us understanding of the possibilities and challenges of utilizing $360^{\circ}$ video in such applications. The long-term collection of feedback has provided us overall understanding of museum goers' attitudes and experiences and also provided us some data on the effects of various upgrades we have introduced to the installations.

The overall feedback to the road grader was positive. The driving was considered fun and the installation was considered something that increases willingness to visit museums. However, the visitors reported that they did not feel like they were actually steering the road grader. Over the revisions, we were able to improve the experience, especially with haptic feedback, but also got some mixed results, particularly with steering mapping adjustment. The rally simulator received overall favorable feedback, it 
was considered fun and the visitors reported that they would have liked to continue using it. While people did not feel like driving the rally car, they reported that they felt like being inside one. The possibility to focus on the different details of a real-life rally car was valued and the pace notes were also considered interesting.

Looking at the user experiences and comments received, we raise immersion, interactivity and additional modalities as the key elements relevant to ODV based museum installations.

\subsection{Immersion}

The immersive nature of the ODV provided genuine value. As earlier work suggests, the HMD based installation seemed to have more beneficial immersion. While the novelty value of a VR headset may play some role in the positive user experience, the objective immersion of the headset is significantly better than the immersion our projector based system could provide. While the horizontal coverage of the projection was close to human field of view, the vertical coverage was much smaller and room floor and ceiling were clearly visible to drivers. This, together with the fact that the simple cabin did not include windows or ceiling limited the immersion. We assume that this is the cause to the rather minimal improvement to the user experience when we added two more projectors to the installation.

In museums, social interaction is often an important part of the experience. From this point of view, HMD based material presentation is not optimal, since it isolates people. It can be argued that, e.g., projection based viewing solutions, which support multiple simultaneous users would be better. In museum settings also issues related to public displays like honey-pot effect can be relevant, overly complex and ambiguous installations may be avoided by visitors.

The downside of the immersion is that immersive solutions are prone to cause simulator sickness, aka, cyber sickness [29]. Especially when VR content, e.g., an ODV is viewed with a HMD, unpredictable camera movements can cause cyber sickness [14]. It is possible to stabilize the video, in particular camera rotations in post-processing. [13] However, shooting videos with a moving camera should always be considered very carefully. Having at least a part of the view stable can reduce unpleasant effects. Our respondents reported minimal ill effects, and while people who know they are prone to cyber sickness likely did not try the installation, the reported numbers are still surprisingly low indicating that the rally simulator was very successful in avoiding cyber sickness. This is probably mostly due to the fact that the rally videos consisted mostly of the fixed car interior, and moving scenery was visible only through car windows. This provided plenty of fixed, clear focus points for the viewers. In addition, the installation contained a couple of minutes of material and the resulting short usage sessions most likely helped as well. Finally, watching the video in the firm cup seats of the rally car provided a stable and comfortable environment. 


\subsection{Interactivity}

In the road grader simulator, we experimented with creating an interactive application with ODV as the primary content. The simulator reacted to accelerator and brake pedals with realistic engine sounds and subtle visual effects. This part of interaction seemed effective. However, the feedback on steering wheel rotation never resulted in adequate feeling of control, as seen in the user experience data. The free form feedback also supports this conclusion. While the users liked that they could "drive" the road grader, they also complained that they could not actually steer it. The fact that inverting the visual steering feedback caused only slight change in the user experience tells that the users did not really feel they were driving a vehicle. Instead, the metaphor for some of the users was steering the world as opposed to steering the camera [36]. At the moment, the installation may appear more interactive than it really is, which can hurt the user experience. We are currently building a solution where more realistic driving simulation controls virtual camera position and heading inside the 3D video sphere with realistic steering model. This solution requires a video, where the vehicle is not visible, a 3D model will be used instead. With these updates we wish to increase the feeling of actually steering the grader, but we acknowledge that we will lose some of the benefits of ODV, namely some of the authenticity and low production costs.

To provide any benefit, a museum installation must first capture the attention of the visitors and intrigue them to interact. The most common use case is that a visitor will use each installation once. Therefore, immediate usability of the installations is critical. Issues studied with public displays, such as the barrier of first use and levels of engagement and related models like audience funnel [19] and honey-pot effect [25] can be valuable tools but at the same time, the interaction must be easy to understand and not intimidate visitors. Utilizing physical elements and genuine items as part of the installations is recommendable in museum settings. These elements can both guide and attract the users and add value to the experience. Both of our installations have received a good number of users and museum personnel consider them worthwhile additions to the exhibits, event when they cause additional maintenance burden. The added value of the physical elements separate the installations from what the visitors could experience already at home and immersion and interactivity separate them from the other exhibits.

\subsection{Additional modalities}

Audio-visual material can benefit from other modalities as well. Haptic sense, both tactile and kinesthetic, were involved in our installations. The real rally car, while interesting to the visitors by itself, as the environment to view the videos added tactility to the experience. Similarly, the grader was interesting, particularly to small children since it allowed them to steer and operate the pedals. The haptic feedback added to the seat in the grader created a statistically significant and noteworthy improvement to the user experience and illustrates how active haptic feedback solutions can provide value. Many of the measures improved with the introduction of this feedback showing that good haptic feedback can improve the overall experience, instead of being just a gimmicky addition. With the use of, potentially authentic, physical props and additional 
feedback modalities museum installations can provide experiences which cannot be found elsewhere.

\section{Conclusions}

Omnidirectional video, aka $360^{\circ}$ video, can be used to efficiently create authentic and immersive experiences and thus has great potential to be utilized in museum installations. Where it is possible to record material related to the exhibit, ODV can provide engaging and entertaining experiences which can also provide valuable information and understanding to the visitors. In this paper, we presented two systems based on ODV material, one viewed with a head-mounted display and the other utilizing a three-projector setup. The installations also utilized physical props and additional modalities. The reported experiences and the analysis of the roles of immersion, interactivity and additional modalities can help others interested in building similar installations. We consider immersion and interactivity to be important factors to help understand the possibilities of ODV based solutions. In museum settings physical elements of the installations can also have an important role.

Authenticity of the presented material is key in museum context and when video capture is an option, ODV can be valuable. In comparison to traditional video, the installations can be more interactive. Even the baseline possibility to control the viewing direction can be appreciated as it allows the visitors to focus on details most interesting to them. Furthermore, the engagement, created via the interactivity and immersion can lead to both user satisfaction and potentially efficient communication of information. Overall, ODV based technology can create entertaining and effective museum installations. The production costs of such installations can also be reasonable; ODV camera solutions are now available at almost all price categories, and programming interactive ODV applications is reasonably easy with tools like Unity3D.

ODV can be especially useful in cases where the immersive nature of ODV is inherently valuable. In our two cases, this value was in (virtually) being inside a special type of vehicle not generally accessible to the public. Similarly, this value may be in a building or a location that is hard to access, or an activity that is rare or dangerous in real life. The user experience data we collected shows that immersive ODV viewing experience can create good and valued experiences. In museum environments, it is often possible to enrich the viewing experience further by adding physical props and some interactivity. In our case, the real rally car, even when it did not match the specific vehicle in videos, nor did the camera position the user's location, enriched the viewing experience. We were also able to minimize cyber sickness in the HMD based installation. In the case of the grader simulator, the realistic seat, especially after the addition of haptic feedback, and the realistic steering wheel and pedals added to the experience.

The limitation of ODV compared to 3D modelled content are the fewer interaction possibilities. ODV based solutions can still provide reasonable level of interactivity and when the installations are properly designed, the authenticity of video content and the reasonable productions costs make them viable options. The museum context best supports short usage sessions and the possibilities of ODV based solutions can efficiently 
fulfill the required level of interactivity. The immersive nature of these solutions can create memorable experiences enhancing the overall museum experience. Respondents to our user experience questionnaires strongly support the idea that these installations can make museums more desirable places to visit.

Acknowledgements. This work was done as a part of MIRACLE project, financed by Tekes - the Finnish Funding Agency for Innovation. We appreciate the involvement and input of Mobilia personnel who participated in the design process, data collection and maintenance of the installations. We thank also Juha Salo and his co-drivers for the possibility to record the materials.

\section{References}

1. Argyriou L, Economou D, Bouki V, Doumanis I (2016) Engaging immersive video consumers: Challenges regarding 360-degree gamified video applications. In: 15th IEEE international conference on ubiquitous computing and communications and 2016 international symposium on cyberspace and security (IUCC-CSS '16). IEEE, pp 145-152. doi:10.1109/IUCC-CSS.2016.028

2. Benko H, Wilson AD (2010) Multi-point interactions with immersive omnidirectional visualizations in a dome. In: ACM international conference on interactive tabletops and surfaces (ITS '10). ACM, New York, NY, USA, pp 19-28. doi:10.1145/1936652.1936657

3. Berning M, Yonezawa T, Riedel T, Nakazawa J, Beigl M, Tokuda H (2013) pARnorama. 360 degree interactive video for augmented reality prototyping. In: the 2013 ACM conference on pervasive and ubiquitous computing (UbiComp '13). ACM, New York, NY, USA, pp 1471-1474. doi:10.1145/2494091.2499570

4. Bleumers L, Van den Broeck W, Lievens B, Pierson J. (2012) Seeing the bigger picture: a user perspective on $360^{\circ} \mathrm{TV}$. In: 10th European conference on interactive TV and video (EuroiTV '12). ACM, New York, NY, USA, pp 115-124. doi:10.1145/2325616.2325640

5. Boult TE (1998) Remote Reality via omnidirectional imaging. In: ACM SIGGRAPH 98 conference abstracts and applications (SIGGRAPH '98). ACM, New York, NY, USA, p 253. doi:10.1145/280953.282215

6. Concert - 360-degree video from ZuZuVideo. https:/www.youtube.com/watch?v=1Kp1_icG328

7. De la Torre F, Vallespi C, Rybski PE, Veloso M, Kanade T. (2005) Omnidirectional video capturing, multiple people tracking and identification for meeting monitoring. Technical report. http://repository.cmu.edu/robotics/128/

8. Decock J, Van Looy J, Bleumers L, Bekaert P (2014) The pleasure of being (there?): an explorative study into the effects of presence and identification on the enjoyment of an interactive theatrical performance using omnidirectional video. AI \& Soc 29(4): 449-459. doi:10.1007/s00146-013-0487-6

9. Fassbender E, Heiden W (2014) Atmosphaeres - $360^{\circ}$ video environments for stress and pain management. In: Ma M, Oliveira MF, Baalsrud Hauge J (eds) Serious games development and applications. LNCS, vol 8778. Springer International Publishing, pp 48-58. doi:10.1007/978-3-319-11623-5

10. Ekola L, Järvinen A (2014) KÄÄNNEKOHTA: 360-videon soveltuminen viittomakielen tulkkausharjoitteluun. Bachelor's thesis. Diaconia University of Applied Sciences, Degree Programme in Sign Language Interpretation. https://publications.theseus.fi/bitstream/handle/10024/73100/Ekola_Jarvinen.pdf?sequence $=1$ 
11. Jennett C, Cox AL, Cairns $P$ (2009) Investigating computer game immersion and the component real world dissociation. In: CHI '09 extended abstracts on human factors in computing systems (CHI EA '09). ACM, New York, NY, USA, pp 3407-3412. doi:10.1145/1520340.1520494

12. Kallioniemi P, Mäkelä V, Saarinen S, Turunen M, Winter Y, Istudor A (2017) User experience and immersion of interactive omnidirectional videos in CAVE systems and headmounted displays. In: Bernhaupt R, Dalvi G, Joshi AK, Balkrishan D, O’Neill J, Winckler M (eds) Human-Computer Interaction - INTERACT 2017. LNCS, vol 10516. Springer, Cham, pp 299-318. doi:10.1007/978-3-319-68059-0_20

13. Kasahara S, Nagai S, Rekimoto J (2015) First person omnidirectional video: system design and implications for immersive experience. In: ACM international conference on interactive experiences for TV and online video (TVX '15). ACM, New York, NY, USA, pp 33-42. doi:10.1145/2745197.2745202

14. Kasahara S, Rekimoto J (2015) JackIn head: immersive visual telepresence system with omnidirectional wearable camera for remote collaboration. In: 21st ACM symposium on virtual reality software and technology (VRST '15). ACM, New York, NY, USA, pp 217-225. doi: $10.1145 / 2821592.2821608$

15. Keskinen, T (2015) Evaluating the user experience of interactive systems in challenging circumstances. Ph.D. thesis. Dissertations in interactive technology 22, University of Tampere. http://urn.fi/URN:ISBN:978-951-44-9972-2

16. Kwiatek K (2012) How to preserve inspirational environments that once surrounded a poet? Immersive $360^{\circ}$ video and the cultural memory of Charles Causley's poetry. In: 18 th international conference on virtual systems and multimedia (VSMM '12). IEEE, pp 243-250. doi:10.1109/VSMM.2012.6365931

17. Kwiatek K, Woolner M (2010) Transporting the viewer into a $360^{\circ}$ heritage story: Panoramic interactive narrative presented on a wrap-around screen. In: 16th international conference on virtual systems and multimedia (VSMM '10). IEEE, pp 234-241. doi:10.1109/VSMM.2010.5665980

18. Mammut \#project360. Home page: https://play.google.com/store/apps/details?id=ch.mammut.project $360 \&$ hl $=$ en

19. Michelis D, Müller J (2011) The Audience Funnel: Observations of gesture based interaction with multiple large displays in a city center. Int J Hum Comput Interact 27(6):562-579. doi:10.1080/10447318.2011.555299

20. Neng LAR, Chambel T (2010) Get around $360^{\circ}$ hypervideo. In: 14th international Academic MindTrek conference: Envisioning Future media environments (MindTrek '10). ACM, New York, NY, USA, pp 119-122. doi:10.1145/1930488.1930512

21. Neumann U, Pintaric T, Rizzo A (2000) Immersive panoramic video. In: 8th ACM international conference on multimedia (MULTIMEDIA 00 ). ACM, New York, NY, USA, pp 493-494. doi:10.1145/354384.376408

22. Okura F, Kanbara M, Yokoya N (2011) Fly-through Heijo palace site: augmented telepresence using aerial omnidirectional videos. In: ACM SIGGRAPH 2011 Posters (SIGGRAPH '11). ACM, New York, NY, USA, Article 78. doi:10.1145/2037715.2037803

23. Onoe Y, Yamazawa K, Takemura H Yokoya N (1998) Telepresence by real-time view-dependent image generation from omnidirectional video streams. Comput Vis Image Underst 71(2):154-165. doi:10.1006/cviu.1998.0705

24. Pakkanen T, Hakulinen J, Jokela T, Rakkolainen I, Kangas J, Piippo P, Raisamo T, Salmimaa M (2017) Interaction with WebVR $360^{\circ}$ video player: Comparing three interaction paradigms. In: 2017 IEEE Virtual Reality (VR). IEEE, pp 279-280. doi:10.1109/VR.2017.7892285 
25. Parra G, De Croon R, Klerkx J, Duval E (2014) Quantifying the interaction stages of a public display campaign in the wild. In: 8th Nordic conference on human-computer interaction: Fun, fast, foundational (NordiCHI '14). ACM, New York, NY, USA, pp 757-760. doi:10.1145/2639189.2639216

26. Peñate W, Pitti CT, Bethencourt JM, de la Fuente J, Gracia R (2008) The effects of a treatment based on the use of virtual reality exposure and cognitive-behavioral therapy applied to patients with agoraphobia. Int J Clin Health Psychol 8(1):5-22.

27. Petry B, Huber J (2015) Towards effective interaction with omnidirectional videos using immersive virtual reality headsets. In: 6th Augmented human international conference (AH '15). ACM, New York, NY, USA, pp 217-218. doi:10.1145/2735711.2735785

28. Ramalho J, Chambel T (2013) Windy sight surfers: sensing and awareness of $360^{\circ}$ immersive videos on the move. In: 11th European conference on interactive TV and video (EuroITV '13). ACM, New York, NY, USA, pp 107-116. doi:10.1145/2465958.2465969

29. Rebenitsch L, Owen C (2016) Review on cybersickness in applications and visual displays. Virtual Reality 20(2):101-125. doi:10.1007/s10055-016-0285-9

30. Rizzo AA, Ghahremani K, Pryor L, Gardner S (2003) Immersive 360-degree panoramic video environments: research on creating useful and usable applications. In: Jacko JA, Stephanidis C (eds) Human-computer interaction: theory and practice, part 1. Lawrence Erlbaum Associates, Mahwah, NJ, USA, pp 1233-1237.

31. Rovelo Ruiz GA, Vanacken D, Luyten K, Abad F, Camahort E (2014) Multi-viewer gesturebased interaction for omnidirectional video. In: 32nd ACM conference on human factors in computing systems (CHI '14). ACM, New York, NY, USA, pp 4077-4086. doi:10.1145/2556288.2557113

32. Saarinen S, Mäkelä V, Kallioniemi P, Hakulinen J, Turunen M (2017) Guidelines for designing interactive omnidirectional video applications. In: Bernhaupt R, Dalvi G, Joshi AK, Balkrishan D, O'Neill J, Winckler M (eds) Human-Computer Interaction - INTERACT 2017. LNCS, vol 10516. Springer, Cham, pp 263-272. doi:10.1007/978-3-319-68059-0_17

33. Slater M (2003) A note on presence terminology. Presence Connect 3(3).

34. Turunen M, Hakulinen J, Hella J, Rajaniemi JP, Melto A, Mäkinen E, Rantala J, Heimonen T, Laivo T, Soronen H, Hansen M, Valkama P, Miettinen T, Raisamo R (2009) Multimodal media center interface based on speech, gestures and haptic feedback. In: Gross T. et al. (eds) Human-Computer Interaction - INTERACT 2009. LNCS, vol 5727. Springer, Berlin, Heidelberg, pp 54-57. doi=10.1007/978-3-642-03658-3_9

35. Turunen M, Hakulinen J, Melto A, Heimonen T, Laivo T, Hella J. (2009) SUXES - user experience evaluation method for spoken and multimodal interaction. In: 10th annual conference of the international speech communication association. ISCA, pp 2567--2570.

36. Ware C, Osborne S (1990) Exploration and virtual camera control in virtual three dimensional environments. In: the 1990 symposium on interactive 3D graphics (I3D '90). ACM, New York, NY, USA, pp 175-183. doi:10.1145/91385.91442

37. Yagi Y (1999) Omnidirectional sensing and its applications. IEICE TRANSACTIONS on Information and Systems E82-D(3):568-579.

38. Zoric G, Barkhuus L, Engström A, Önnevall E (2013) Panoramic video: Design challenges and implications for content interaction. In: 11th European conference on interactive TV and video. ACM, New York, NY, USA, pp 153-162. doi:10.1145/2465958.2465959

39. Zoric G, Engström A, Barkhuus L, Ruiz-Hidalgo J, Kochale A. (2013) Gesture interaction with rich TV content in the social setting. In: Exploring and enhancing the user experience for television, Workshop of ACM SIGCHI conference on human factors in computing systems (CHI'13). 